\title{
acueducto de Tempul
}

\author{
E. TORROJA
}

emplazamiento: Jerez de la Frontera

(Cádiz)

propietario: Sociedad de Aguas

aก̃o: 1925

constructora: Hidrocivil

$535-1$

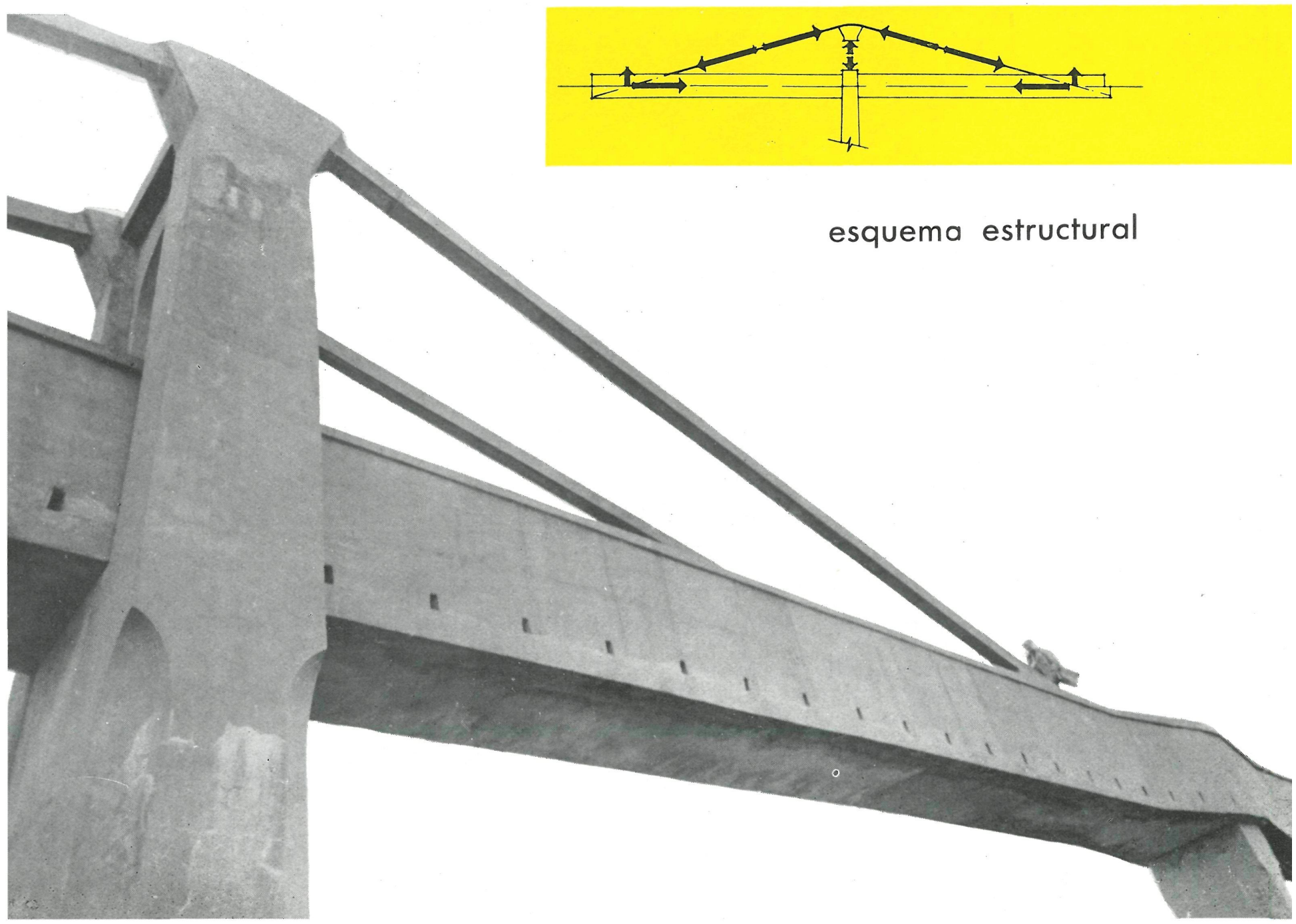



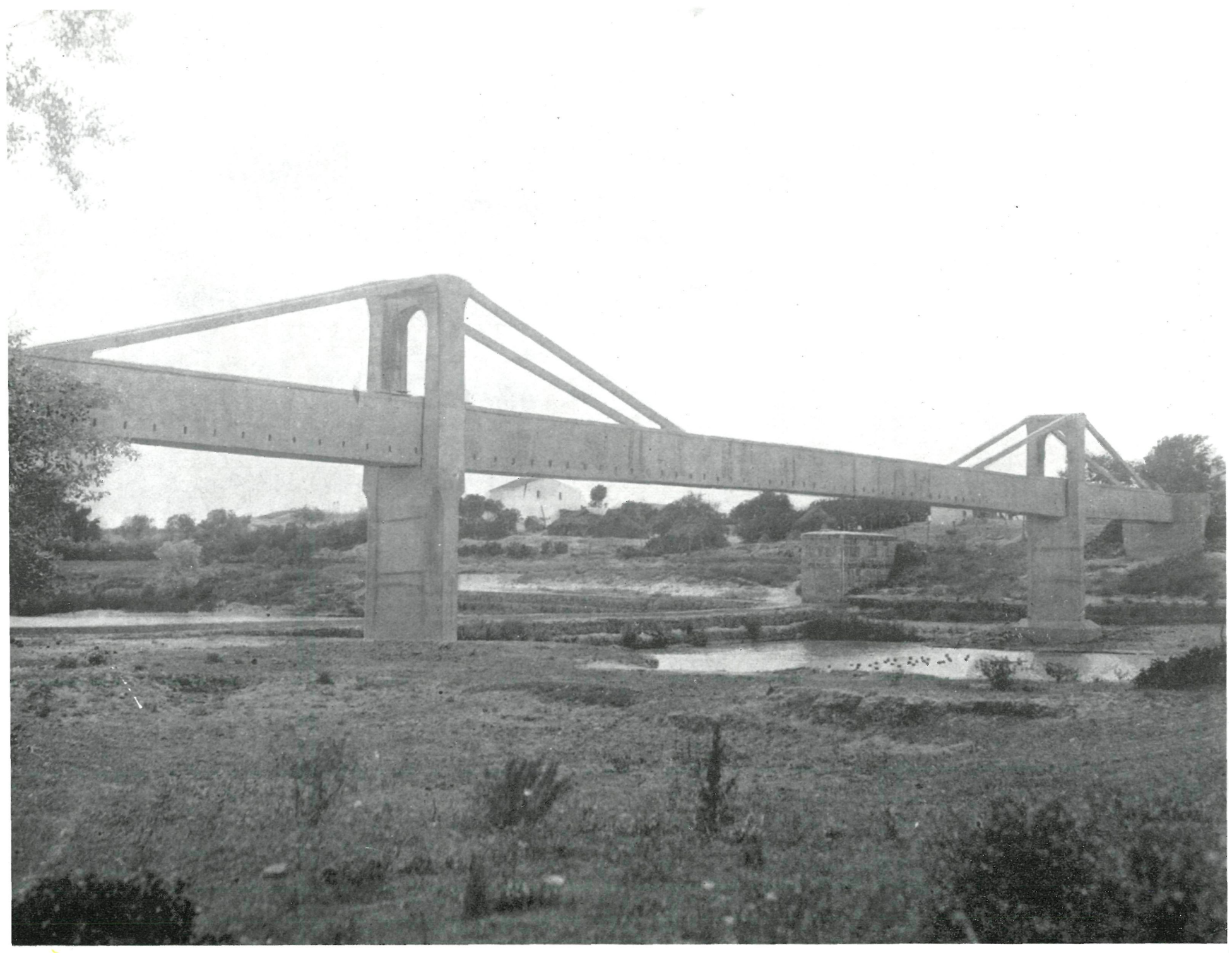

Los habitantes de Jerez de la Frontera-la vieja ciudad española que produce los nobles vinos Xerez-, necesitan también beber agua, y el abastecimiento de la población requería un acueducto para cruzar el cauce del Guadalete.

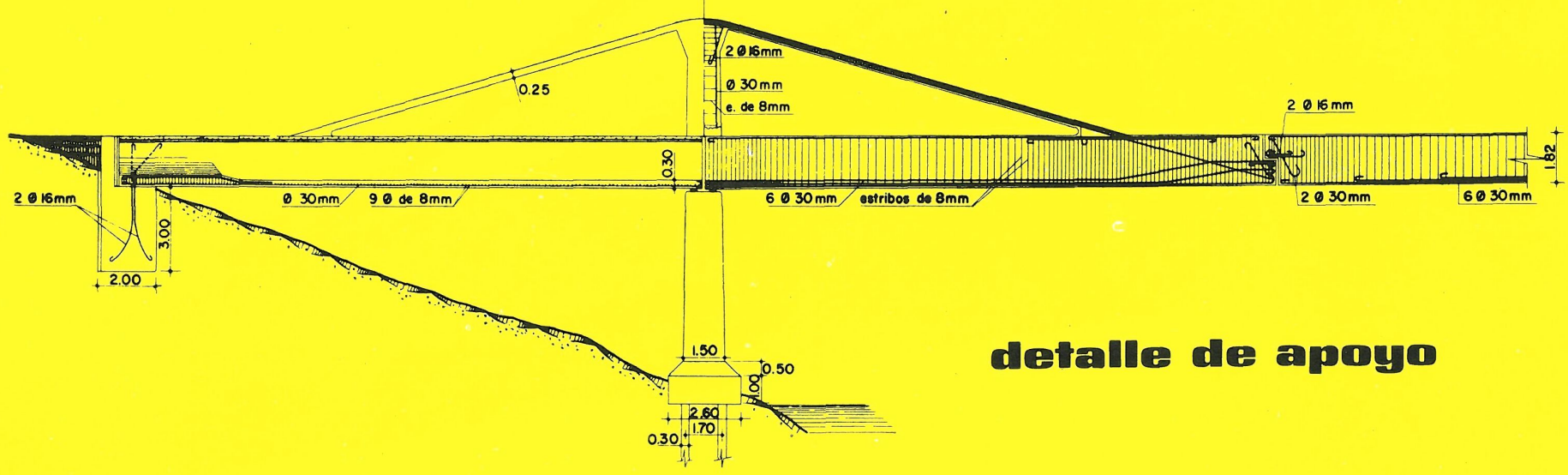


Un primer proyecto estaba formado por catorce tramos de 20 metros de luz sobre pilas cimentadas a 4 metros de profundidad en la parte cubierta solamente por las grandes avenidas, y con cimentaciones sobre pilotes de 10 metros en los tres tramos cubiertos normalmente por las aguas.

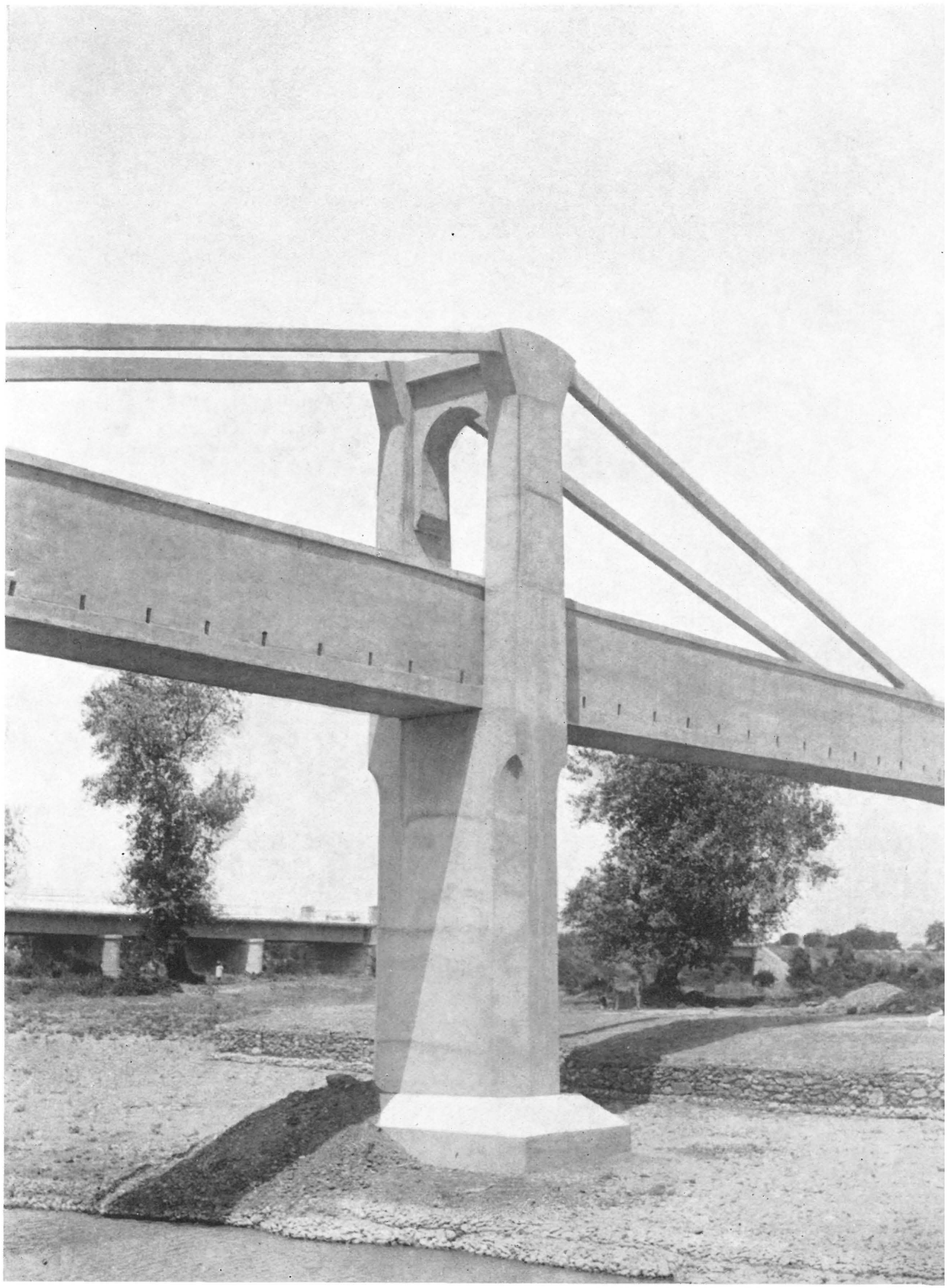




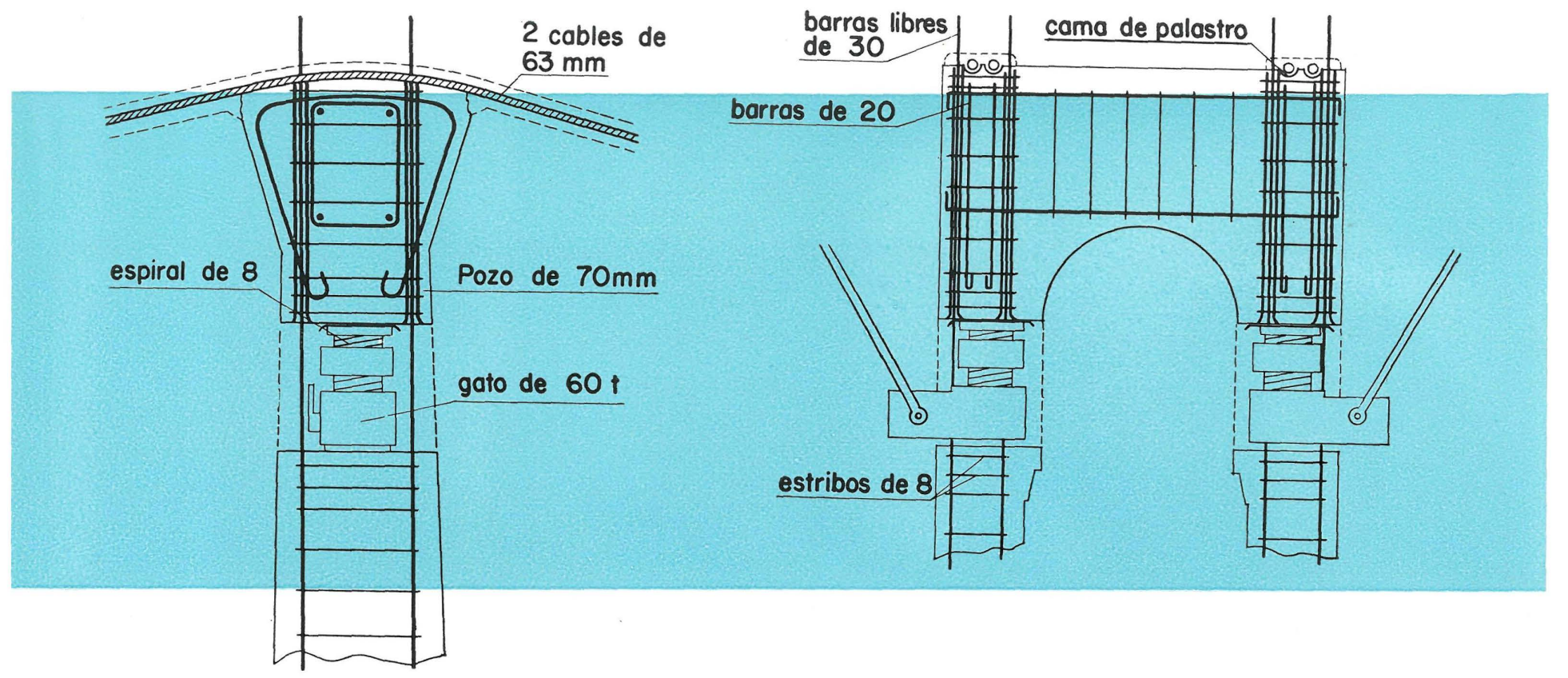

\section{gatos de elevación del castillete}

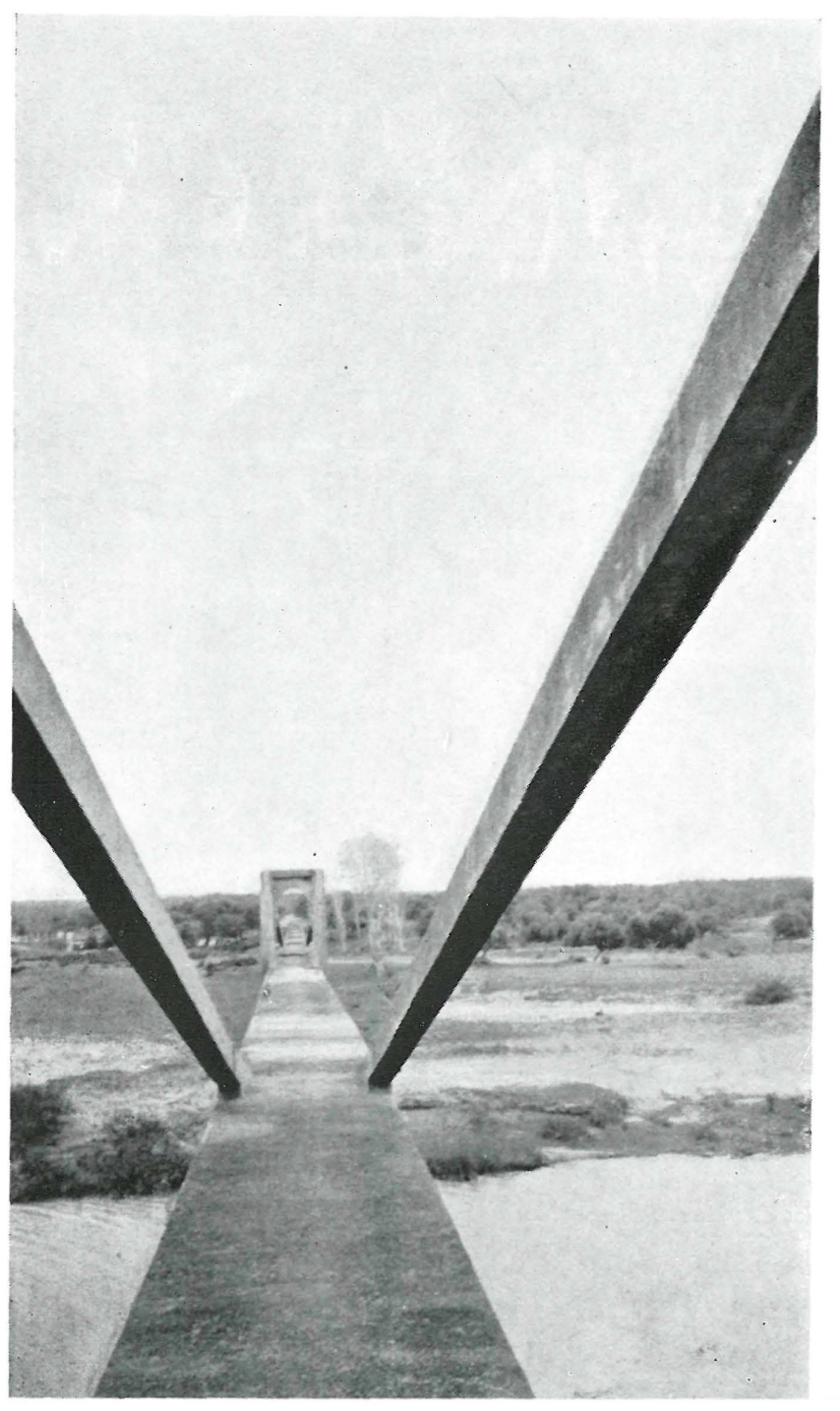

De esta manera se evitaban los costosos agotamientos que hubieran exigido estas últimas cimentaciones si se hubiese intentado hacerlos por pozos bajo el agua. Los tramos son de sección en cajón para alojar en su interior las tuberías de la conducción.

Presentado el proyecto, la Sociedad requirió la supresión de las dos pilas que caían sobre la parte más profunda del cauce, por temor a que la mayor profundidad de las aguas en esa zona pudiese socavar esas cimentaciones. Acuciado por la premura de tiempo y deseoso de resolver el problema rápidamente, alterando lo menos posible al proyecto ya estudiado, ideé mantener los mismos tramos, sustituyendo la acción sustentadora de esas dos pilas por unos tirantes que, pasando por encima de las pilas laterales convenientemente sobreelevadas, fueran a anclarse en los extremos de los tramos contiguos. De este modo, bastaba reforzar las cimentaciones de esas pilas, trasladando a ellas los pilotes de las pilas suprimidas; y lo único que había que calcular era la sección de los tirantes, cuyo esfuerzo podía obtenerse en pocos minutos por una simple descomposición vectorial de las cargas que actuaban sobre las pilas suprimidas. 


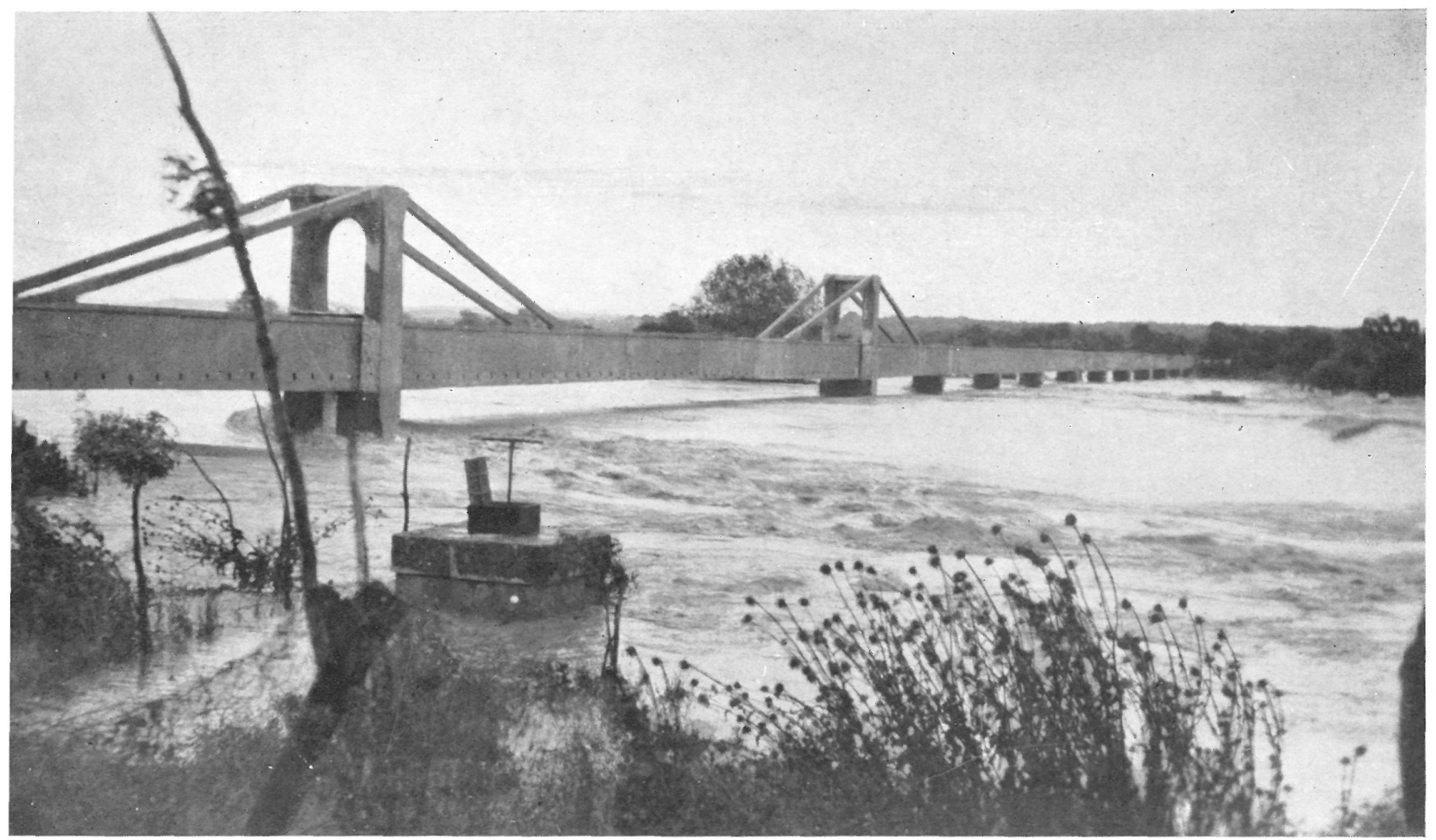

La solución permitía hacer la obra en pocos meses durante el estiaje, y fue rápidamente aceptada, con lo que se dio comienzo inmediatamente a la construcción.

Pero los tirantes, con sus anclajes, resultaban de unos 50 metros de longitud. En 1926 no existía la técnica del pretensado, y la soldadura no estaba tampoco suficientemente desarrollada para confiar a ella una obra de esta importancia. La solución clásica de empalmar barras por solape dentro del hormigón, tampoco satisfacía. Me propuse, por consiguiente, emplear cables de alambres trenzados de acero de alta calidad. De este modo podía disponer rápidamente de los tirantes de una sola pieza en toda su longitud. Su transporte, enrollándolos en grandes tambores como es costumbre, era fácil, y su desenrollamiento y colocación en obra resultaban también cómodos y económicos.

Quedaban únicamente por resolver los problemas derivados del gran alargamiento que normalmente toman estos cables al ponerles en tensión por primera vez. La solución consistió en montarlos pasando por encima de las cabezas o partes superiores de las pilas, dejando éstas separadas de los fustes. Con esto, una vez hormigonados los tramos con los extremos de los cables anclados en ellos, podía hacerse subir las cabezas de las pilas, mediante gatos hidráulicos, tendiendo a aumentar la longitud de los tirantes hasta ponerlos en su debida tensión de trabajo. En efecto, justamente cuando el hormigonado de todos los tramos estaba terminado y el hormigón había acabado de alcanzar su necesaria resistencia, una fuerte avenida amenazó con arrastrar la cimentación de madera; pero bastó accionar los gatos, bajo las cabezas de las pilas, para que, al subir éstas unos $25 \mathrm{~cm}$, los cables alcanzasen la oportuna tensión y levantasen los tramos centrales $(5 \mathrm{~cm})$, despegándolos de su cimbra, que quedó a merced del río.

Pasados unos días y hecha la sobrecarga total de los tramos, se hormigonaron los espacios que quedaban entre las pilas y sus cabezas, se retiraron los gatos, se completó el hormigonado de los huecos que ocupaban, y se envolvieron los cables, también en hormigón, para evitar todo peligro de oxidación. 
La sección transversal es en cajón, con paredes de $15 \mathrm{~cm}$ de espesor. Los tirantes están formados por dobles cables de acero, de 37 hilos, de $3 \mathrm{~mm}$, trabajando a 27 kilogramos por milímetro cuadrado.

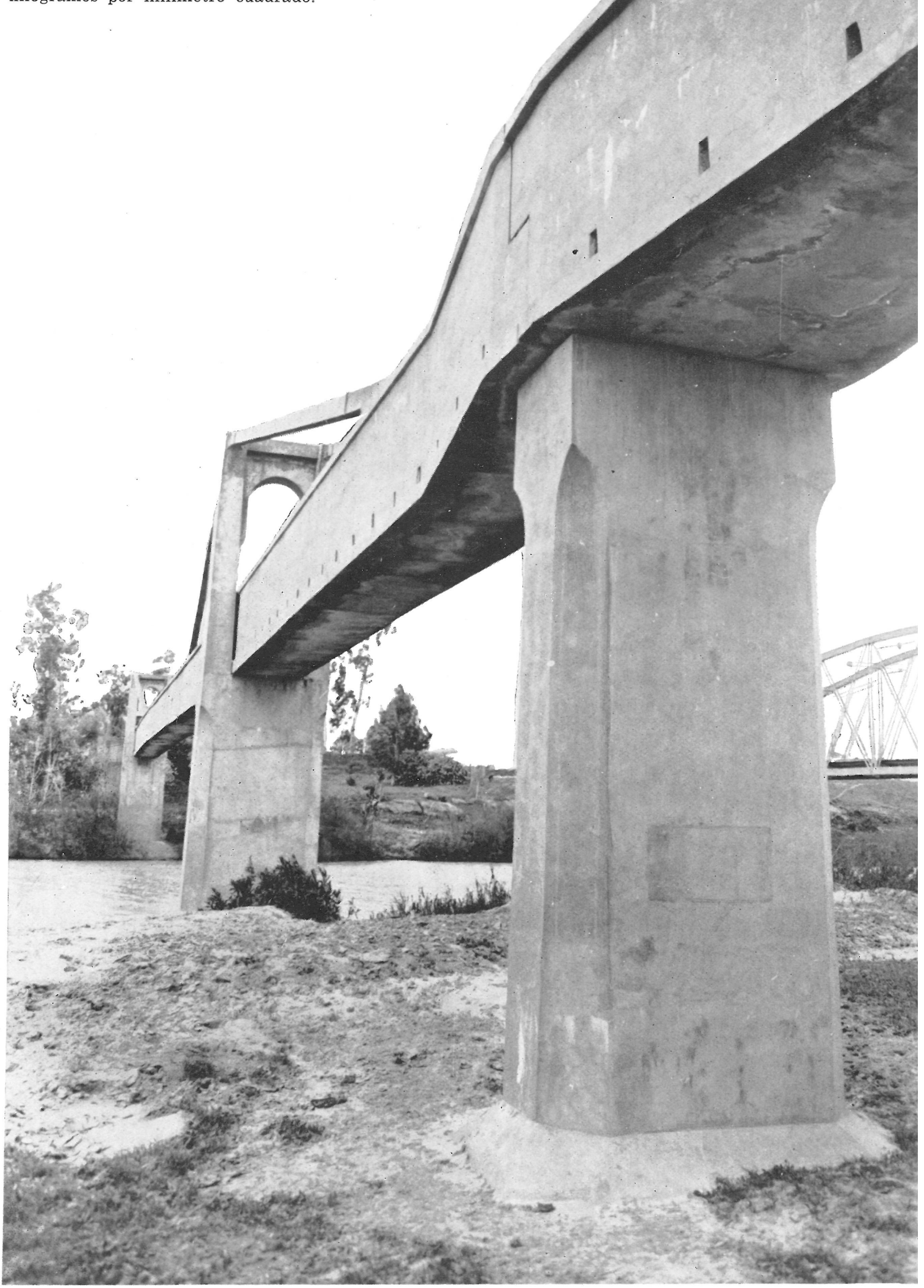

